\title{
Formation Mechanism of Nanostructures on the Stainless Steel Surface by Femtosecond Laser Pulses
}

\author{
Md. Shamim Ahsan ${ }^{* 1, * 2}$, Yeong Gyu Kim ${ }^{* 1}$, and Man Seop Lee ${ }^{* 1}$ \\ ${ }^{*}$ Photonics Application Lab, Korea Advanced Institute of Science and Technology (KAIST), 335 \\ Gwahak-ro, Yuseong-gu, Daejeon 305-701, South Korea \\ ${ }^{* 2}$ Electronics and Communication Engineering Discipline, School of Science, Engineering and \\ Technology, Khulna University, Khulna-9208, Bangladesh \\ E-mail:shamim@kaist.ac.kr
}

In this paper, we report on the formation of a variety of micro/nano-metric structures on the stainless steel surfaces by single-beam direct femtosecond laser writing. Periodic microgratings of different line widths and period are fabricated on the sample surfaces. Random nanostructures are observed inside the microgratings, when laser pulses with low laser energies are applied on the samples. Unlike random nanostructures, periodic nanostructures are produced inside the microgratings, when laser pulses with high average pulse energy are applied. Besides, self-organized nanogratings, with variable periods, are formed all over the micro-machined area on the stainless steel surfaces due to the overlap of the microgratings, where the orientation of the nanogratings is parallel to the incident light polarization. Finally, we illustrate the formation mechanism of various kinds of nanostructures, produced on the stainless steel surfaces during our experiment. DOI:10.2961/jlmn.2012.02.0007

Keywords: Femtosecond Laser, single beam direct laser writing technique, microgratings, selforganized nanogratings, nanostructures

\section{Introduction}

In the last few decades, femtosecond laser based processing of various materials has gained the attention of many researchers. In recent time, femtosecond lasers have been considered as a promising tool in micro/nanomachining of diverse metals [1-10]. Researchers have already reported the micro/nano-structuring of various metals using several femtosecond laser based technologies: holographic technology [11] and near-field enhancement induced by self-assembled Particle Lens Array (PLA) [12]. However, holographic technology is complicated due to the requirement of the obligatory coincidence of multiple laser beams. On the counterpart, PLA based technology is limited to surface micro/nano-machining only, because this technology can't produce structures inside the samples. Due to the limitations of other available technologies, Single Beam Direct Laser Writing (SBDLW) technology comes to the forefront of the research field.

Using SBDLW technology, researchers reported the formation of a variety of micro/nano-metric structures on different material surfaces. Interaction of femtosecond laser pulses with various metals and the formation of Laser Induced Periodic Surface Structures (LIPSS) or Nanostructure Covered Laser Induced Periodic Surface Structures (NC-LIPSS) on various metal surfaces have been studied comprehensively in some recent studies [1-5]. Researchers have also reported the absorption [6] and the enhanced energy coupling [7-9] on diverse metal surfaces by producing a variety of micro/nano-scale features. Colorizing stainless steel surfaces by femtosecond laser induced self-organized nanogratings (LIPSS) or periodic microholes have also been reported [10]. Due to the complexity involved in the formation of nanostructures (LIPSS/NC-LIPSS) on metal surfaces by femtosecond lasers, researchers have proposed quite a few different models in explaining the formation mechanism of LIPSS/NC-LIPSS: interference of the highintensity laser beam with the laser induced plasma waves [13] and Coulomb explosion [14]. Although a lot of studies have been reported so far on femtosecond laser-metal interactions and the formation of LIPSS/NC-LIPSS on different metals, the information regarding the interactions of femtosecond laser pulses with stainless steel surfaces is still inadequate. In addition, the formation mechanism of the laser induced nanostructures on stainless steel surfaces needed more clarification. Thus, it is important to examine the formation of various laser induced nanostructures on stainless steel surfaces and their formation mechanism.

In this paper, using SBDLW technology, we produced long distance (we fabricated $1 \mathrm{~mm}$ long) horizontal microgratings on the stainless steel surfaces by controlling the irradiation conditions of a femtosecond laser beam. The period of the microgratings are varied from $10 \mu \mathrm{m}$ to 100 $\mu \mathrm{m}$. Self-organized nanogratings are produced all over the laser micromachined area, when two or more horizontal microlines are overlapped. The period of the subwavelength self-organized nanogratings are ranging from $474 \mathrm{~nm}$ to $596 \mathrm{~nm}$. Periodic nanostructures, other than selforganized nanogratings, are evident inside the microgratings at high average pulse energy of the laser pulses $(1.3 \mu \mathrm{J})$ with the scanning speed of $1 \mathrm{~mm} / \mathrm{s}$. At low laser energies (varied from $437 \mathrm{~nJ}$ to $538 \mathrm{~nJ}$ ), random nanostructures are formed inside the microgratings. The experimental results clearly suggest the certain dependence of the nanostructures' patterns on various irradiation conditions of the femtosecond laser beam such as the average pulse energy, the scanning speed, and the scanning step. Above all, 
we describe briefly the formation mechanism of the micro/nano-metric structures, produced on the stainless steel surfaces during the experiment.

\section{Experimental Details}

In order to carry out our experiment, we used a Ti:sapphire femtosecond laser. The central wavelength of the laser beam, the pulse width, and the pulse repetition rate were $786 \mathrm{~nm}, 183 \mathrm{fs}$, and $1 \mathrm{kHz}$, respectively. The laser beam was focused perpendicularly on the top of the sample surfaces through a $100 \times$ achromatic objective lens, which has a numerical aperture of $\mathrm{NA}=0.7$. The photograph of the experimental setup is shown in Fig. 1.
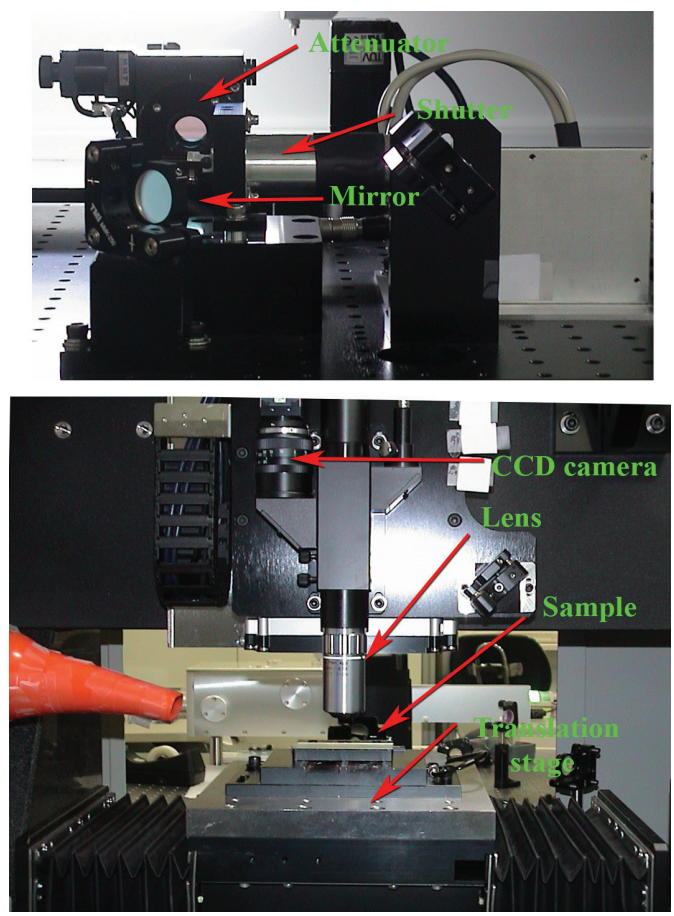

Fig. 1 Photograph of the experimental setup for the fabrication of a variety of micro/nano-structures on the stainless steel surfaces.

Stainless steel samples, with a thickness of $100 \mu \mathrm{m}$ and a complex refractive index of $\tilde{\eta}=\eta+i k=3.15+i 3.07$ at $786 \mathrm{~nm}$, were used in the experiment. The samples were mounted on a computer-controlled 3 axis (x, y, and $\mathrm{z}$ ) linear translation stage. The resolution of the translation stage was $100 \mathrm{~nm}$. To control the exposure time of the laser beam, we placed a high-speed computer-controlled mechanical shutter in the path of the laser beam. A computer-controlled circular attenuator was placed after the mechanical shutter to control the power of the femtosecond laser pulses. A linear polarizer (s-polarized) was placed in the optical path, after the attenuator, to produce polarized laser pulses. To measure the average power of the femtosecond laser pulses after the objective lens, we used a power meter. In order to guide the laser beam towards the sample surfaces, a number of fully reflective mirrors were placed in the optical path.

In order to fabricate periodic micro/nano-gratings on the stainless steel surfaces, the average pulse energy, the scanning speed of the laser beam, and the scanning step of the horizontal lines were varied from $341 \mathrm{~nJ}$ to $1.3 \mu \mathrm{J}, 0.5$ $\mathrm{mm} / \mathrm{s}$ to $2 \mathrm{~mm} / \mathrm{s}$, and $500 \mathrm{~nm}$ to $100 \mu \mathrm{m}$, respectively. When the scanning step was low, two or more micro-lines were overlapped and consequently self-organized nanogratings were formed all over the micro-machined area. To analyze the morphology of the micro/nano-metric features, produced on the stainless steel surfaces, we examined the micro/nano-structured samples under a Scanning Electron Microscope (SEM). To remove the contamination from the sample surfaces, the stainless steel samples were cleaned by $99.3 \%$ methyl alcohol before the laser micromachining and SEM examination.

\section{Results}

Using SBDLW technology, we fabricated periodic microgratings, of different periods and line widths, on the stainless steel surfaces by controlling the irradiation conditions of a femtosecond laser beam. Due to the reduction of the scanning step, two or more horizontal microlines are overlapped. As a consequence, self-organized nanogratings were formed on the sample surfaces. Unlike self-organized nanogratings, periodic nanostructures were observed inside the microgratings at high average pulse energy. On the contrary, random nanostructures are evident inside the microgratings at low laser energies. In this section, we will explain, in details, about the fabricated micro/nano-metric structures, on the sample surfaces, and their formation mechanism.

\subsection{Formation of periodic microgratings}

We shone the laser beam perpendicularly onto the sample surfaces in order to form the periodic microgratings. The laser beam was scanned in the horizontal direction to fabricate long distance horizontal periodic microgratings (we fabricated $1 \mathrm{~mm}$ long; any distance is possible), as shown in Fig. 2.

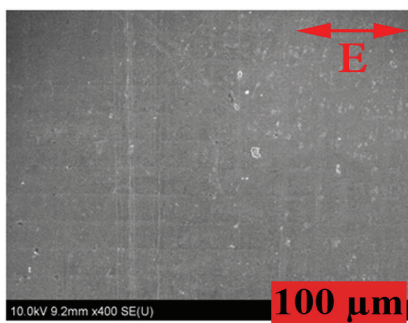

(a)

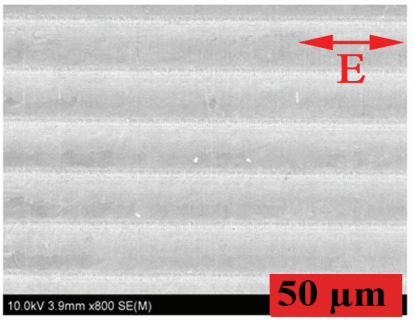

(c)

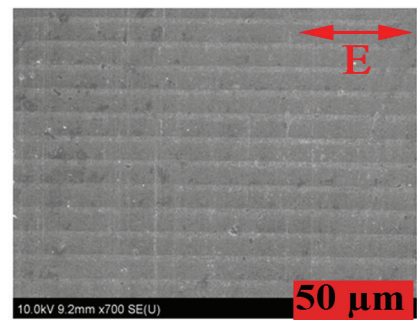

(b)

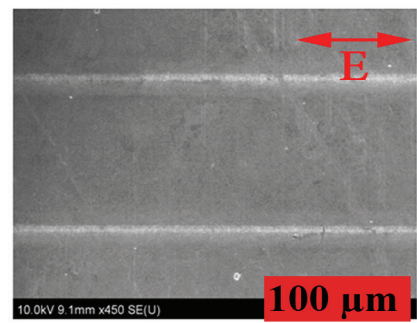

(d)
Fig. 2 SEM images of the fabricated periodic microgratings on the stainless steel surfaces at a scanning speed of 1 $\mathrm{mm} / \mathrm{s}$ ( $\mathbf{E}$ denotes the direction of electric field vector). (a) At average pulse energy $(E)$ of $437 \mathrm{~nJ}$ and scanning step (h) of $20 \mu \mathrm{m}$; (b) at $E=538 \mathrm{~nJ}$ and $h=10 \mu \mathrm{m}$; (c) at $E=$ $1.3 \mu \mathrm{J}$ and $h=20 \mu \mathrm{m}$; (d) at $E=01.3 \mu \mathrm{J}$ and $h=100 \mu \mathrm{m}$. 
Due to the low scanning speed of the translation stage, two or more laser pulses were overlapped, and as a result microgratings were formed on the stainless steel surfaces. The period of the linear microgratings were controlled by the scanning step, which was varied from $10 \mu \mathrm{m}$ to $100 \mu \mathrm{m}$. The SEM images of Fig. 2 indicate that the line widths of the microgratings followed increasing trend with the increase of the average pulse energy (varied from $437 \mathrm{~nJ}$ to $1.3 \mu \mathrm{J})$. The line widths of the microgratings were ranging from $2.75 \mu \mathrm{m}$ to $9 \mu \mathrm{m}$.

\subsection{Formation of self-organized periodic nanogratings}

In order to produce self-organized nanogratings, we scanned the laser beam perpendicularly on the sample surfaces in the horizontal direction. The average pulse energy of the laser beam, the scanning speed of the translation stage, and the scanning step of the horizontal lines were varied from $341 \mathrm{~nJ}$ to $437 \mathrm{~nJ}, 0.5 \mathrm{~mm} / \mathrm{s}$ to $2 \mathrm{~mm} / \mathrm{s}$, and 500 $\mathrm{nm}$ to $2 \mu \mathrm{m}$, respectively. Due to the low scanning step, two or more microlines were overlapped. As a result, selforganized nanogratings were printed on the stainless steel surfaces. The self-formed nanogratings were oriented in the parallel direction of the laser polarization direction. The SEM images of the self-organized nanogratings are shown in Fig. 3.

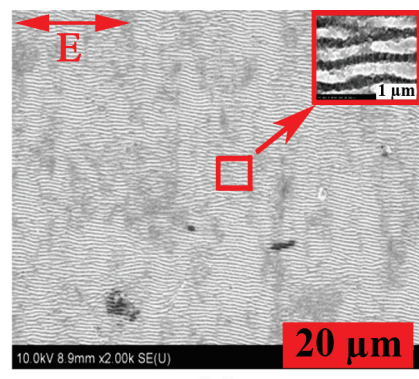

(a)

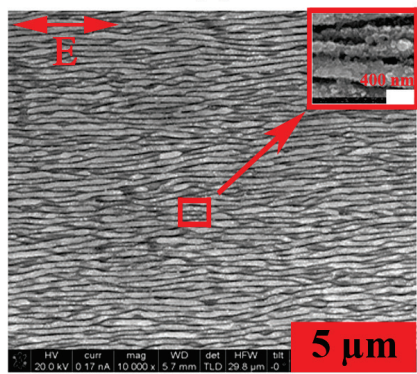

(c)

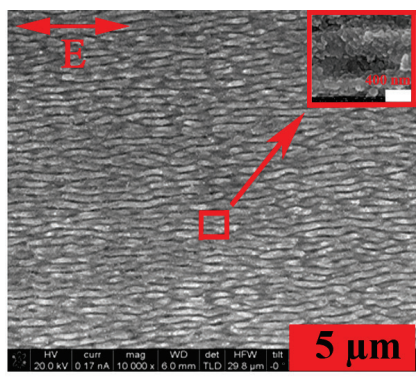

(b)

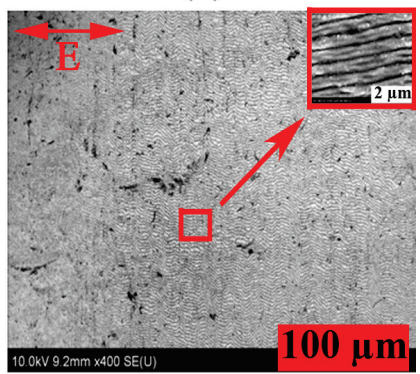

(d)
Fig. 3 SEM images of the self-organized nanogratings produced on the stainless steel surfaces ( $\mathbf{E}$ denotes the direction of electric field vector). (a) At average pulse energy $(E)$ of $341 \mathrm{~nJ}$, scanning speed $(v)$ of $0.5 \mathrm{~mm} / \mathrm{s}$, and scanning step $(h)$ of $1 \mu \mathrm{m}$; (b) at $E=361 \mathrm{~nJ}, v=2 \mathrm{~mm} / \mathrm{s}$, and $h$ $=2 \mu \mathrm{m}$; (c) at $E=427 \mathrm{~nJ}, v=2 \mathrm{~mm} / \mathrm{s}$, and $h=2 \mu \mathrm{m}$; (d) at $E=437 \mathrm{~nJ}, v=1 \mathrm{~mm} / \mathrm{s}$, and $h=500 \mathrm{~nm}$.

With the increase of the average pulse energy (varied from $341 \mathrm{~nJ}$ to $437 \mathrm{~nJ}$ ), the line widths of the microgratings were increased (ranging in the range of $2 \mu \mathrm{m}$ to $2.75 \mu \mathrm{m}$ ), as expected. During the formation of nanogratings, the line widths of the micro-lines were higher than the scanning steps of the horizontal micro-lines (varied from $500 \mathrm{~nm}$ to
$2 \mu \mathrm{m})$. As a result, two or more micro-lines were overlapped and consequently self-organized nanogratings were printed on the stainless steel surfaces. From the magnified SEM images of Fig. 3, it is evident that the nanogratings' period followed increasing trend with the increase of the average pulse energy and vice versa. The average value of the nanogratings' period of Figs. 3(a, b, c, and d) are 474 $\mathrm{nm}, 580 \mathrm{~nm}, 587 \mathrm{~nm}$, and $596 \mathrm{~nm}$, respectively.

Randomly oriented spherical-shaped nanostructures, with a diameter ranging from $10 \mathrm{~nm}$ to $60 \mathrm{~nm}$, were also observed on the top of and inside the self-organized nanogratings as shown in the magnified SEM images of Fig. 3. This kind of nanostructures was independent of the laser energy since they were evident irrespective of the laser energy and the number of overlapped micro-lines.

\subsection{Formation of random nanostructures}

We also fabricated periodic microgratings at low laser energies (varied from $437 \mathrm{~nJ}$ to $538 \mathrm{~nJ}$ ) with gratings' period varying from $10 \mu \mathrm{m}$ to $50 \mu \mathrm{m}$ as illustrated in Fig. 4 .

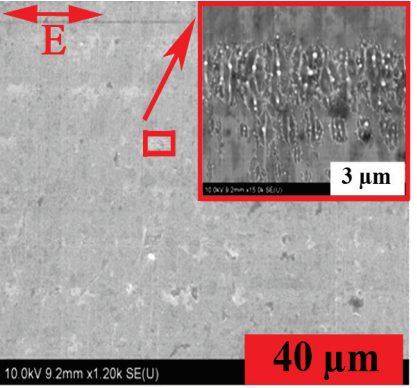

(a)

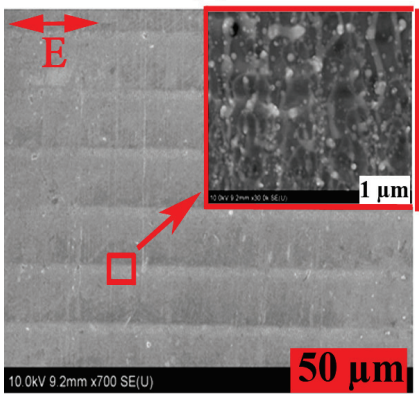

(c)

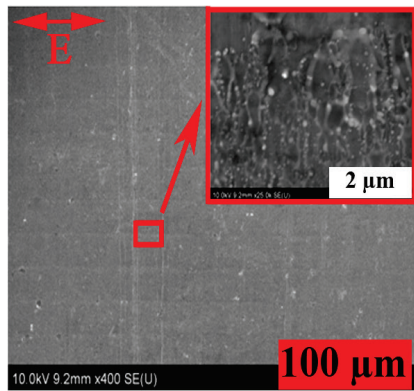

(b)

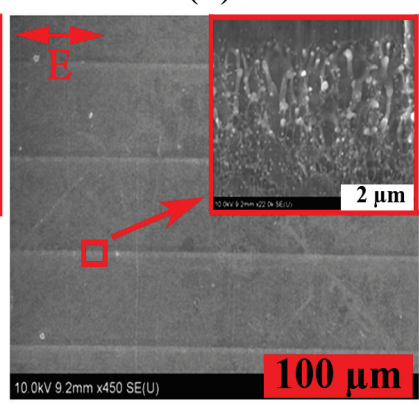

(d)
Fig. 4 SEM Images of the random nanostructures produced inside the microgratings on the stainless steel surfaces at a scanning speed of $1 \mathrm{~mm} / \mathrm{s}$ (E denotes the direction of electric field vector). (a) At average pulse energy $(E)$ of $437 \mathrm{~nJ}$ and scanning step $(h)$ of $10 \mu \mathrm{m}$; (b) at $E=437 \mathrm{~nJ}$ and $h=$ $20 \mu \mathrm{m}$; (c) at $E=538 \mathrm{~nJ}$ and $h=20 \mu \mathrm{m}$; (d) at $E=538 \mathrm{~nJ}$ and $h=50 \mu \mathrm{m}$.

Random nanostructures, distributed in a scattered manner, were evident inside the microgratings. The SEM images of the random nanostructures, produced under different laser energies and scanning steps are shown in the magnified SEM images of Fig. 4. The scanning speed of the translation stage was set at $1 \mathrm{~mm} / \mathrm{s}$. The line widths of the microgratings were $\sim 4 \mu \mathrm{m}$ (for $437 \mathrm{~nJ}$ ) and $\sim 6 \mu \mathrm{m}$ (for $538 \mathrm{~nJ})$. The random nanostructures were irregular in shape and increased with the increase of the average pulse energy. 


\subsection{Formation of periodic nanostructures}

We also fabricated long period horizontal microgratings with gratings' period varying from $50 \mu \mathrm{m}$ to $100 \mu \mathrm{m}$ at the average pulse energy of $1.3 \mu \mathrm{J}$ and the scanning speed of 1 $\mathrm{mm} / \mathrm{s}$. The SEM images of the periodic microgratings and the periodic nanostructures inside the microgratings are depicted in Fig. 5. The line widths of the microgratings in both cases were $\sim 9 \mu \mathrm{m}$ as shown in Figs. 5(a and c).

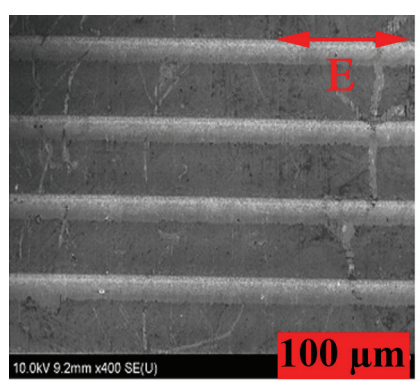

(a)

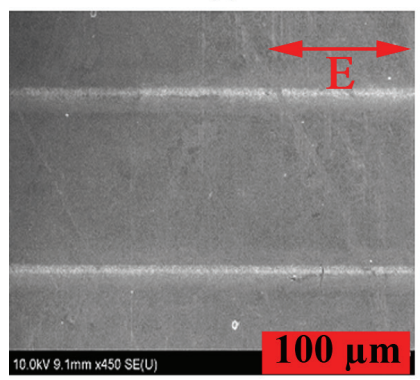

(c)

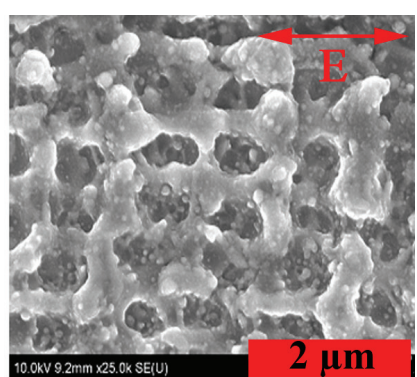

(b)

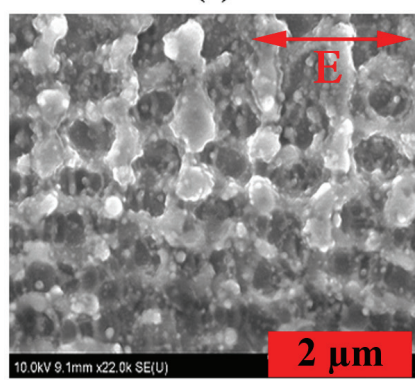

(d)
Fig. 5 SEM Images of the periodic nanostructures formed inside the microgratings on the stainless steel surfaces, produced at average pulse energy of $1.3 \mu \mathrm{J}$ and a scanning speed of $1 \mathrm{~mm} / \mathrm{s}(\mathbf{E}$ denotes the direction of electric field vector). (a) Periodic microgratings with gratings' period of $50 \mu \mathrm{m}$; (b) magnified SEM image of the periodic nanostructures, produced inside the microgratings of Fig. $5(\mathrm{a})$; (c) periodic microgratings with gratings' period of $100 \mu \mathrm{m}$; (d) magnified SEM image of the periodic nanostructures, produced inside the microgratings of Fig. 5(c).

Unlike self-organized nanogratings or random nanostructures, periodic nanostructures were evident inside the microgratings. The period of the periodic nanostructures for both cases was $\sim 1 \mu \mathrm{m}$ in the horizontal direction and $\sim 650 \mathrm{~nm}$ in the vertical direction. The SEM images of the periodic nanostructures are shown in Figs. 5(b and d). The randomly oriented spherical-shaped nanostructures, with a diameter ranging from $10 \mathrm{~nm}$ to $60 \mathrm{~nm}$, were also observed on the top of and inside the periodic nanostructures of Figs. 5( $b$ and $d)$.

\section{Discussion}

In this paper, we demonstrate the formation of periodic microgratings on the stainless steel surfaces. From the experimental results of Fig. 2, it is certain that the line widths of the microgratings follow the increasing trend with the increase of the average pulse energy, which is in reasonable agreement with the model proposed by A. P. Joglekar et. al. [15]. Due to the increase of the focal spot area of the laser beam with the increase of the laser energy, the line widths of the microgratings also increase. Microgratings' period, on the other hand, is controlled by the scanning step of the horizontals lines. We fabricated $1 \mathrm{~mm}$ long microgratings; however, any length is possible.

When the scanning steps of the horizontal microlines are set less than the line widths of the horizontal microlines, microlines are overlapped in the horizontal direction. As a result, self-organized nanogratings are formed on the sample surfaces as shown in Fig. 3. The formation mechanism of the self-organized nanogratings can be explained using the following interpretation.

When femtosecond laser pulses are applied on the stainless steel surface, plasma waves are formed. The Selforganized nanogratings of Fig. 3 are formed due to the interaction of the high-intensity laser beam and the plasma waves produced on the sample surfaces. The femtosecond laser beam-metal interactions undergo several courses of actions: melting \& re-solidification of a surface region, photochemical spallation of single or multiple layers or droplets, and phase explosion [16]. Beyond certain threshold energy, phase explosion takes place, where the ablation depths can fluctuate with constant laser fluence. This process is considered as the primary mechanism for the printing of nanogratings on the sample surfaces.

As mentioned earlier, the self-organized nanogratings, produced during our experiments, have variable periods showing the dependence on various laser parameters such as the average pulse energy and the number of laser pulses.

There are several models in explaining the nanogratings' period. Shimotsuma et. al. [17] proposed one model, where the nanogratings' period is a function of the laser fluence (average pulse energy per unit area) and the number of laser pulses. The nanogratings' period is defined by the following equation [17].

$$
d=\frac{2 \pi}{\sqrt{\frac{1}{T_{e}}\left(\frac{m_{e} \omega^{2}}{3 k_{B}}-\frac{e^{2} N_{e}}{3 \varepsilon_{0} k_{B}}\right)-k_{p h}^{2}}}
$$

where $d$ is the grating period, $T_{\mathrm{e}}$ is the electron temperature, $m_{\mathrm{e}}$ is the electron mass, $\omega$ is the angular frequency, $k_{\mathrm{B}}$ is the Boltzmann's constant, $e$ is the electron charge, $N_{\mathrm{e}}$ is the electron density, $\varepsilon_{0}$ is the dielectric constant of air, $\mathrm{k}_{\mathrm{ph}}=$ $\omega \eta / c$ is the wave vector, $\eta$ is the refractive index of materi$\mathrm{al}$, and $c$ is the speed of light.

From Eq. (1), it is evident that the nanogratings' period increases sharply with the increase of the electron concentration, i.e., the laser fluence. However, this model is applicable for the nanogratings produced inside glass materials. For metals, Eq. (1) can provide imaginary period, which is unrealistic.

According to the Bonch-Bruevich et. al. model [18], the period of the LIPSS, i.e., self-organized nanogratings, formed by linearly polarized laser pulses, can be obtained by the equation as follows:

$$
d=\frac{\lambda}{\eta \pm \sin \theta} \text { with } \mathbf{g} \| \mathbf{E}
$$

where $d$ is the nanogratings' period, $\lambda$ is the wavelength of the incident laser beam, $\eta$ is the real part of the refractive index of the material, $\theta$ is the incident angle of the laser 
beam on the sample surface, $\mathbf{g}$ is the grating vector, and $\mathbf{E}$ is the electric field vector of the incident wave. The highspatial-frequency structure (HSFS), produced on titanium sample follows the model represented by Eq. (2) [19]. By using Eq. (2), the expected period of the self-organized nanogratings in our case is either $189 \mathrm{~nm}$ or $366 \mathrm{~nm}$, which are quite different than the nanogratings' period obtained during our experiment. The complex dielectric constant can be written as [20]

$$
\begin{aligned}
& \tilde{\varepsilon}(\omega)=\varepsilon_{I}(\omega)+i \varepsilon_{2}(\omega)=(\eta+i k)^{2} \\
& \varepsilon_{l}(\omega)=\eta^{2}-k^{2}=\varepsilon_{l, \text { inter }}-\frac{\omega_{p}^{2} \tau^{2}}{1+\omega^{2} \tau^{2}} \\
& \varepsilon_{2}(\omega)=2 \eta k=\frac{\omega_{p}^{2} \tau}{\omega\left(1+\omega^{2} \tau^{2}\right)}
\end{aligned}
$$

where $\tilde{\varepsilon}(\omega)$ is the complex dielectric function with real component of $\varepsilon_{l}(\omega)$ and imaginary component of $\varepsilon_{2}(\omega)$, $\omega$ is the angular frequency of the incident laser beam, $\omega_{P}$ is the plasma frequency, and $\tau$ is the relaxation time of the free electron. For unmodified stainless steel sample, the complex dielectric function is $\tilde{\varepsilon}(\omega)=0.498+i 19.34$. However, the value of the real and imaginary components of the dielectric constant is solely dependent on the plasma frequency and the relaxation time of the free electrons. The relaxation time of the electrons $(\tau)$ decreases with the increase of the electron temperature $\left(T_{e}\right)$ caused by the heating of the electron subsystem $\left(\tau \propto 1 / T_{e}^{2}\right)$, which also increase the plasma frequency $\omega_{P}$ [21]. The plasma frequency $\omega_{P}$ is a function of the real part of the refractive index, which can be defined as follows [20]:

$$
\omega_{P}^{2}=\frac{N_{e} e^{2}}{\varepsilon_{0} m}
$$

where $N_{e}$ is the electron density, $e$ is the electron's charge, $\varepsilon_{0}$ is the dielectric constant of vacuum, and $m$ is the effective optical mass of electron. Since, the self-organized nanogratings are formed due to the overlap of multiple pulses (number of pulses are variable) at the same spot, the dielectric constant of the metal surface is altered during the experiment due to the variation of $\tau$ and $\omega_{P}$. As a result, the refractive index of the material is changed as well. If we consider the grating period of $596 \mathrm{~nm}$ (Fig. 3(d)), we obtain the value of the real part of refractive index is either 0.31 or 2.31, which are different than the unmodified stainless steel sample. Depending on the number of overlapped pulses or the laser energy, the electron temperature $T_{e}$, as well as $\tau$ and $\omega_{P}$ are varied, which in turn cause the variation of the refractive index with the number of overlapped laser pulses and the laser energy. These phenomena influence the variation of nanogratings' period with the laser energy and the number of applied laser pulses.

In order to explain the dependence of the nanogratings' period on the average pulse energy, we divide the focal spot of the Gaussian laser pulses into two regions: region 1 is for high laser energy (central part) and region 2 is for low laser energy (surroundings) as shown in Figs 6(a).

Self-organized nanogratings can be formed either by applying several pulses at the same spot or by overlapping several micro-lines. When several pulses are applied at the same spot, long period nanogratings are formed at region 1 , whereas, short period nanogratings are formed at region 2 , as shown in Fig. 6(b).

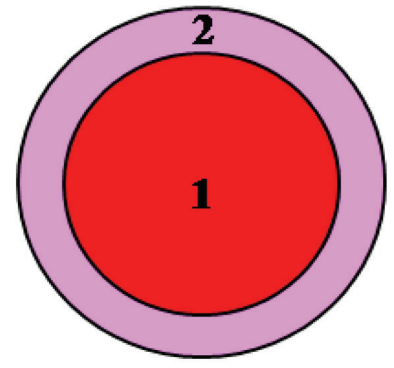

(a)

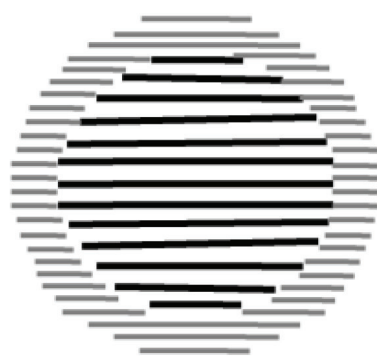

(b)

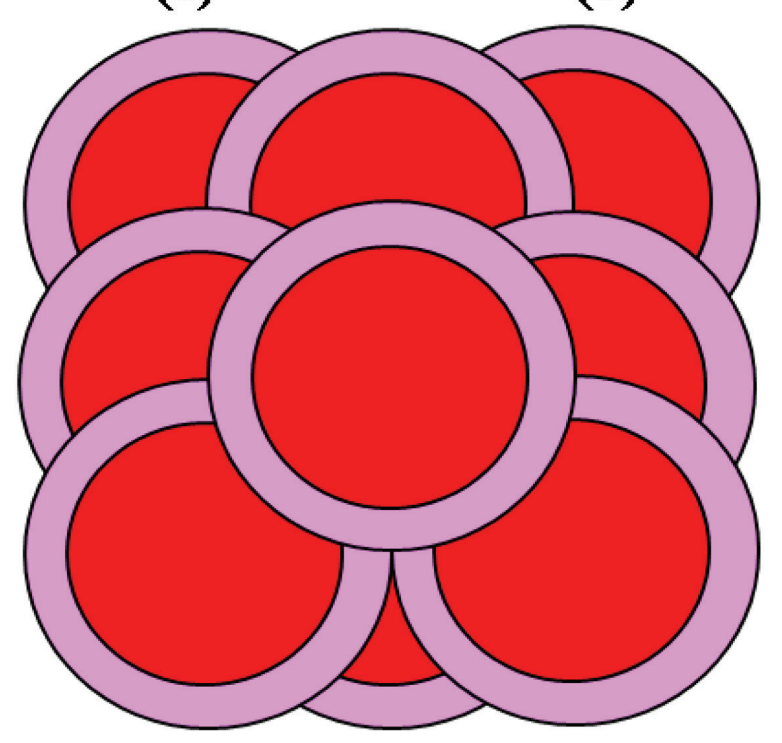

(c)

Fig. 6 Schematic diagram for the formation of nanogratings due to overlapped pulses. (a) Focal spot of single laser pulse; (b) formation of nanogratings after several pulses are applied at the same spot; (c) overlap of several pulses in both horizontal and vertical directions.

Although there is no defined threshold energy for the two regions of Fig. 6(a), there is clear evidence that the self-formed nanogratings' period are quite different in different regions of the focal spot [22]. Due to the overlap of the micro-lines, femtosecond laser pulses are overlapped in both horizontal and vertical directions (not at the same spot) and self-organized nanogratings with gratings' period in the sub-wavelength range are produced on the sample surfaces, as shown in Fig. 6(c).

Although the laser energy applied for the nanogratings of Fig. 3(b) is slightly higher than the laser energy applied for the nanogratings of Fig. 3(a), the grating period of Fig. 3(a) is significantly lower than that of Fig. 3(b). Due to the low scanning speed and the short scanning step, more pulses are overlapped (in both horizontal and vertical directions) in Fig. 3(a) compared to Fig. 3(b), which drastically reduces the grating period of Fig. 3(a). Due to the overlap of more laser pulses cause the increase in $T_{e}$. As a result, the value of the electrons' relaxation time $\tau$ decreases. Since, $\omega>\omega_{P}$, the value of the real part of the refractive 
index $\eta$ increases, which consecutively reduce the nanogratings' period of Fig. 3(a).

As mentioned before, the direction of the self-organized nanogratings is parallel to the laser polarization direction. Since the formation of nanogratings is dependent on the interference of the incident laser beam and the plasma waves (which are influenced by the laser polarization), it is usual that the nanogratings' direction is also influenced by the laser polarization direction.

Furthermore, at low laser energies, random nanostructures are produced inside the microgratings, rather than forming self-organized nanogratings as shown in Fig. 4. From the experimental results, it is evident that the overlap of the laser pulses only in the horizontal direction might not be enough to produce self-organized nanogratings on the stainless steel surfaces.

Unlike self-organized nanogratings or random nanostructures, periodic nanostructures are formed inside the microgratings, when high laser energy $(1.3 \mu \mathrm{J})$ is applied as shown in Fig. 5. This kind of periodic nanostructures are similar to the NC-LIPSS reported by Vorobyev et. al., formed after the application of 30 pulses at the same spot [5]. The period of the nanostructures are $\sim 1 \mu \mathrm{m}$ in the horizontal direction and $\sim 650 \mathrm{~nm}$ in the vertical direction. The period of the periodic nanostructures in the horizontal direction is equal to $\sim 1 \mu \mathrm{m}$, which is equal to the center to center distance of the laser pulses; at a pulse repetition rate of $1 \mathrm{kHz}$ and scanning speed of $1 \mathrm{~mm} / \mathrm{s}$, the center to center distance of the laser pulses is expected to be $\sim 1 \mu \mathrm{m}$. However, the reason behind the period of the periodic nanostructures in the vertical direction $(\sim 650 \mathrm{~nm})$ is undiscovered yet; multiple peaks of the laser beam may be responsible behind this phenomenon.

It is noteworthy to mention here that similar kinds of periodic microgratings are fabricated in both Figs. 4 and 5. The only difference is the average pulse energy. However, the nanostructures produced inside the microgratings of Fig. 4 are randomly oriented, which structures are certainly different from the periodic nanostructures produced in Fig. 5. As a result, we can infer that the high laser energy of the Gaussian shape beam might be responsible for the production of periodic nanostructures.

Furthermore, the randomly distributed spherical- shaped nanostructures, with diameters ranging in the range of 10 $\mathrm{nm}$ to $60 \mathrm{~nm}$, are formed on the top of and inside the micro/nano-gratings, produced on the stainless steel surfaces. Unlike periodic or random nanostructures, spherical-shaped nanostructures are independent of the average pulse energy. These nanostructures might have re-deposited during laser machining.

\section{Conclusion}

We demonstrated the fabrication of a variety of micro/nano-scale features on the stainless steel surfaces using SBDLW technology. Periodic microgratings of different line widths were produced on the sample surfaces by varying different parameters of a femtosecond laser beam. Self organized nanogratings were produced all over the sample areas when two or more microlines were overlapped due to the shorter scanning step. More importantly, the dependence of the nanogratings' period on the average pulse energy and the number of applied laser pulses were discovered.
Inside the periodic microgratings, random nanostructures were produced at low laser energies. However, periodic nanostructures, dissimilar to the self-organized nanogratings or the random nanostructures were formed inside the microgratings at high laser energy. Randomly oriented spherical-shaped nanostructures were also formed on the top of and inside the micro/nano-gratings irrespective of the average pulse energy. A systematic study under diverse experimental conditions clearly suggests the certain dependence of the nanostructures' patterns on various laser parameters, especially, on the average pulse energy. Above all, we illustrated the formation mechanism of the reported micro/nano-metric structures on the stainless steel surfaces. We strongly believe that our research work will be able to play important roles in learning the interactions of femtosecond laser pulses with stainless steel surface and exemplifying a variety of femtosecond laser induced micro/nano-structures on stainless steel surface.

\section{Acknowledgements}

This work was supported by the Brain Korea 21 Project, the School of Information Technology, KAIST in 2012.

\section{References}

[1] B. K. Nayak, M. C. Gupta and K. W. Kolasinski: Appl. Phys. A, 90, (2008) 399.

[2] J. Wang and C. Guo: Appl. Phys. Lett., 87, (2005) 251914.

[3] A. Y. Vorobyev and C. Guo: Appl. Surf. Sci., 253, (2007) 7272.

[4] A. Y. Vorobyev and C. Guo: Opt. Express, 14, (2006) 2164.

[5] A. Y. Vorobyev and C. Guo: Appl. Phys. A, 86, (2007) 321.

[6] S. E. Kirkwood, Y. Y. Tsui, R. Fedosejevs, A. V. Brantov and V. Y. Bychenkov: Phys. Rev. B, 79, (2009) 144120.

[7] A. Y. Vorobyev and C. Guo: Opt. Express, 14, (2006) 13113.

[8] A. Y. Vorobyev and C. Guo: Appl. Phys. Lett., 86, (2005) 011916.

[9] A. Y. Vorobyev, V. M. Kuzmichev, N. G. Kokody, P. Kohns, J. Dai and C. Guo: Appl. Phys. A, 82, (2006) 357.

[10] M. S. Ahsan, F. Ahmed, Y. G. Kim, M. S. Lee and M. B. G. Jun: Appl. Surf. Sci., 257(2011) 7771.

[11] Vass, K. Osvay and B. Hopp: Opt. Express, 14, (2006) 8354.

[12] Y. Zhou, M. H. Hong, J. Y. H. Fuh, L. Lu, B. S. Lukyanchuk and Z. B. Wang: J. Alloys Compd., 449, (2008) 246.

[13] A. Y. Vorobyev, V. S. Makin and C. Guo: J. Appl. Phys., 101, (2007) 034903.

[14] S. Sakabe, S. Shimuzu, M. Hashida, F. Sato, T. Tsuyukushi, K. Nishihara, S. Okihara, T. kagawa, Y. Izawa, K. Imasaki and T. Iida: Phys. Rev. A, 69, (2004) 023203.

[15] A. P. Joglekar, H. Liu, G. J. Spooner, E. Meyhöfer, G. Mourou and A. J. Hunt: Appl. Phys. B, 77, (2003) 25.

[16] L. V. Zhigilei, Z. Lin and D. S. Ivanov: J. Phys. Chem. C, 113, (2009) 11892. 
[17] Y. Shimotsuma, P. G. Kazansky, J. Qiu and K. Hirao: Phys. Rev. Lett., 91, (2003) 247405.

[18] A. M. Bonch-Bruevich, M. N. Libenson, V. S. Makin, and V. V. Trubaev: Opt. Engg., 31, (1992) 718.

[19] Y. Huang, S. Liu, W. Li, Y. Liu, and W. Yang: Opt. Express, 17 (2009) 20756.

[20] S. A. Maier: "Plasmonics: Fundamentals and Applications" (Springer, USA, 2007) p. 10.

[21] Z. Lin, L. V. Zhigilei: Phys. Rev. B, 77 (2008) 075133 .
[22] L. Qi, K. Nishii, Y. Namba: Opt. Lett., 34 (2009) 1846.

(Received: May 12, 2011, Accepted: March 29, 2012) 